\title{
L'âge et ses usages sexués sur les sites de rencontres en France (années 2000)
}

Age and its gendered uses in online dating sites in France (2000-present)

\section{Marie Bergström}

\section{(2) OpenEdition}

12 Journals

\section{Édition électronique}

URL : http://journals.openedition.org/clio/12789

DOI : 10.4000/clio.12789

ISSN : 1777-5299

Éditeur

Belin

\section{Édition imprimée}

Date de publication : 1 décembre 2015

Pagination : 125-146

ISBN : 9782701194325

ISSN : 1252-7017

Référence électronique

Marie Bergström, "L'âge et ses usages sexués sur les sites de rencontres en France (années 2000) », Clio. Femmes, Genre, Histoire [En ligne], 42 | 2015, mis en ligne le 01 décembre 2018, consulté le 14 mai 2020. URL : http://journals.openedition.org/clio/12789; DOI : https://doi.org/10.4000/clio.12789 


\section{L'âge et ses usages sexués sur les sites de rencontres en France (années 2000)}

L'âge structure les rencontres amoureuses. Les couples en témoignent par leur caractère à la fois endogame et asymétrique. Ils réunissent souvent des partenaires proches en âge et, dans le cas des couples hétérosexuels, placent généralement l'homme dans la position de l'aîné. Quelle signification l'âge a-t-il donc dans la sélection des partenaires? À cette question, l'observation de la composition des unions n'apporte pas de réponse: elle révèle le produit final mais reste silencieuse sur son mode de production. Comprendre la manière dont se forment les couples, et le rôle de l'âge dans ce processus, implique de s'intéresser aux rencontres et aux jugements amoureux qui s'y opèrent.

Pour cette étude, on s'intéresse à un type de relations en particulier; celles qui se nouent sur les sites de rencontres sur Internet (cf. en fin d'article "Méthodes et données»). Ces sites, dédiés à l'appariement de partenaires intimes, se sont largement diffusés en France depuis le début des années 2000ํ․ Ils élargissent ainsi la géographie amoureuse et sexuelle mais changent en même temps l'organisation des rencontres. Alors que celles-ci commencent habituellement par une rencontre physique, les usagers des sites se découvrent par le biais de "profils d'utilisateurs" avant de se connaître de visu. Ce nouveau scénario modifie la place de l'âge dans la sélection des partenaires. Il donne le primat à l'âge civil plutôt qu'à l'âge

* L'auteure remercie la société Meetic qui lui a fourni une partie des données mobilisées dans ce texte. Elle tient aussi à remercier le programme OxPo (Oxford - Sciences Po) pour le soutien financier qu'il lui a accordé l'année où cette recherche a été réalisée.

1 Bozon 2008 : 276-277 ; Bergström 2014 : 86-91. 
social qui prévaut lors des interactions en face-à-face. L'article revient sur cette réorganisation des rencontres sur Internet. Plus précisément, il s'en sert comme un miroir des rencontres «ordinaires ». Parce que les sites changent le rôle de l'âge, ils révèlent d'autant mieux comment cette propriété opère dans l'appariement des partenaires.

Par le biais des sites de rencontres, l'objectif est donc d'éclairer la signification de l'âge dans la formation des couples, en ligne mais aussi hors ligne. La démonstration procède en trois temps. Un bref retour sur l'histoire et l'organisation des sites montre d'abord la manière dont ils changent le scénario de la rencontre. L'étude des pratiques de présentation de soi souligne ensuite la traduction difficile de l'âge subjectif en termes chronologiques. Enfin, l'analyse des aspirations amoureuses des utilisateurs montre les usages sexués de l'âge et revient sur les inégalités de genre dans la formation des couples.

\section{Le scénario inversé des rencontres médiatisées}

Les sites de rencontres sur Internet sont une invention récente. Ils apparaissent aux États-Unis au milieu des années 1990 avant d'être importés en France quelques années plus tard. En tant que services commerciaux dédiés à la mise en relation de partenaires amoureux et sexuels, ces sites s'inscrivent cependant dans une histoire plus longue. Ils se situent dans la lignée des agences et des annonces matrimoniales qui se développent à la fin du XIX ${ }^{e}$ siècle. Les agences, qui se multiplient à partir des années 1870, ciblent une classe bourgeoise aisée et urbaine à qui elles assurent des rencontres matrimoniales en toute discrétion et dont elles reçoivent une commission sur la dot en cas de conclusion heureuse ${ }^{2}$. Les annonces, qui se diffusent avec l'essor de la presse écrite au tournant du $\mathrm{XX}^{\mathrm{e}}$ siècle, touchent des cercles sociaux plus larges ${ }^{3}$. Un des principaux supports en est le Chasseur français qui publie ses premières annonces matrimoniales dans les années 1880 et dont la rubrique "mariages » prend son envol dans la période d'entre-deux-guerres ${ }^{4}$. L'usage de ces

\footnotetext{
2 Kalifa 2011.

3 Garden 2008 [1981] : 288.

$4 \quad$ Martin 1980 : 299 ; Garden 2008 [1981] : 289.
} 
dispositifs commerciaux peine cependant à entrer dans les mœurs. Objets d'une forte critique dès leur origine ${ }^{5}$, ils représentent une pratique marginale qui, au milieu des années 1980, concerne moins de $2 \%$ des Français ${ }^{6}$.

Les sites de rencontres ont, eux, connu un certain succès. En 2013, environ 14\% de la population française, âgée de 25 ans à 65 ans, s'était déjà inscrit sur un tel site. Inédits par leur forte diffusion, ces sites ne s'inscrivent pas moins dans la continuité des services de rencontres antérieurs dont notamment les annonces matrimoniales. Cet héritage est d'abord manifeste chez les concepteurs : les premiers sites de rencontres étatsuniens sont, à l'origine, des entreprises spécialisées dans les petites annonces ${ }^{7}$. La continuité est aussi visible dans l'architecture des sites qui se basent en premier lieu sur des fiches auto-descriptives où les usagers se présentent et détaillent le partenaire souhaité. Ces fiches font l'objet d'un exercice d'écriture particulier, présent dans les premières annonces matrimoniales ${ }^{8}$, qui se retrouve sur les sites de rencontres où les fiches prennent également le nom d'« annonces».

Enfin, ces sites reposent sur un scénario de rencontres semblable aux services matrimoniaux précédents et qui les distingue fortement des modes de rencontres ordinaires. Un élément important de ce scénario est le report de la rencontre physique à un deuxième temps. Sur les sites, comme lors du recours aux annonces et aux agences, les partenaires se découvrent d'abord «sur le papier» avant de se voir « en vrai ». Il s'agit d'un renversement important du processus de rencontre qui relativise le corps comme support des premières impressions. Alors que l'apparence physique constitue habituellement la première source d'information sur l'interlocuteur, les « indicateurs naturels » de l'identité sociale sont ici remplacés par des indices écrits9. Les rencontres médiatisées se caractérisent ainsi par un jugement processuel des partenaires

5 Kalifa 2011.

6 Source : Enquête Les Situations familiales, Ined, 1985. Champ: individus vivant en France et âgés de 21 ans à 44 ans (N : 4091).

7 Bergström $2014: 72$.

8 Garden 2008 [1981] : 290.

9 Singly $1984: 524$. 
potentiels. Ces derniers sont tout d'abord évalués sur la base de leurs propriétés objectives (âge, situation matrimoniale, parentalité, profession, niveau d'éducation, etc.) pour ensuite être appréciés selon leur manière d'incorporer ces caractéristiques (hexis corporelle, habits, manières de parler et de bouger $)^{10}$.

Le rôle de l'âge dans la sélection amoureuse se trouve par conséquent modifié. Dans des contextes d'interaction en face-à-face, l'âge est une caractéristique autant sociale que biologique. Alors qu'en apparence il s'agit d'une propriété objective, il prend en fait un sens différent selon les contextes et les acteurs ${ }^{11}$. C'est ainsi que telle personne, de par son comportement, son habillement ou sa situation professionnelle, sera considérée comme « jeune», alors que telle autre, de même âge mais d'allure et de statut différents, sera regardée comme quelqu'un de 《mature ${ }^{12}$. Cette perception sociale de l'âge se perd en partie lors des rencontres médiatisées où il se manifeste en termes chiffrés, d'une part, et se trouve détaché des autres propriétés de la personne, d'autre part. Les services de rencontres procèdent ainsi à une objectivation de l'âge en le présentant sous sa forme chronologique.

De même, ces services font de l'âge un élément central de la sélection des partenaires. C'est tout particulièrement le cas sur les sites de rencontres où l'indication de son sexe et de son âge, de même que des préférences relatives au sexe et à l'âge du partenaire, constituent souvent des informations obligatoires pour pouvoir s'inscrire. Si les usagers sont par ailleurs libres de choisir les éléments à mettre en avant, ces informations doivent être renseignées pour pouvoir valider le profil d'utilisateur et accéder au site. L'âge est donc un composant fondamental à la fois de l'autoportrait et de la recherche de partenaires. C'est d'autant plus le cas qu'il sert à filtrer les membres du site. N'ayant ni la possibilité ni la volonté d'explorer l'ensemble des profils présentés, les utilisateurs procèdent en effet à un tri à l'aide d'un moteur de recherche. L'âge est un élément central dans cette procédure de présélection. Il constitue un critère discriminant qui réduit d'emblée le vivier des partenaires potentiels.

\footnotetext{
10 Bergström $2014: 274$.

11 Bourdieu 1980.

12 Bozon 1990b : 583.
} 
Par rapport aux rencontres en face-à-face, les rencontres en ligne modifient donc à la fois la nature et la place de l'âge. Elles tendent à réduire celui-ci à son caractère chronologique, d'une part, et en font une condition d'éligibilité des partenaires, d'autre part. Cette organisation des rencontres en ligne les rapproche du processus de recrutement professionnel. Face à un nombre souvent abondant de candidatures, les employeurs procèdent à une présélection par étapes. La première consiste justement à évaluer les candidats «sur profil» - CV et lettres de motivation - afin de convoquer seulement un petit nombre en entretien ${ }^{13}$. D'une manière semblable, les usagers des sites de rencontres réduisent d'abord l'éventail des partenaires à l'aide de critères de recherche standardisés. Ils procèdent à une opération de « qualification » ${ }^{14}$ des candidats en établissant l'éligibilité de ces derniers à partir d'un nombre restreint de critères, dont l'âge en premier lieu.

Ce nouveau rôle donné à l'âge dans la sélection amoureuse n'est pas sans susciter de réactions. Si l'organisation des sites modifie indéniablement les logiques d'évaluation des partenaires, les usagers ne s'y plient pas entièrement. Cela se voit dans la présentation de soi où les utilisateurs déploient des stratégies pour contourner le caractère réducteur de l'âge civil. Cela est également visible dans les préférences d'âge déclarées qui, loin de se référer simplement à un âge chronologique, reflètent les significations diverses accordées à cette caractéristique du partenaire. Précisément parce qu’ils tentent de réduire l'âge à un critère uniforme, les sites font ressortir son caractère éminemment social et sexué.

\section{Traduire l'âge en chiffres : les stratégies de présentation de soi}

Contrairement aux annonces matrimoniales - où les usagers se présentent en texte libre et choisissent alors d'indiquer ou non leur âge $^{15}$ - les sites de rencontres comportent un champ obligatoire relatif à

13 Eymard-Duvernay \& Marchal $2000: 412,416$.

14 Eymard-Duvernay \& Marchal 1997 ; Chaulet 2009.

15 Parmi les annonces matrimoniales du Chasseur français, publiées entre 1978 et 1979 et étudiées par François de Singly, environ 20\% sont sans indication quant à l'âge de l'annonceur. Singly 1984. 
la date de naissance. Les réponses fournies révèlent des usages détournés de cette question qui peuvent être compris comme une réticence vis-à-vis de son caractère à la fois obligatoire et contraignant. Un de ces usages consiste à indiquer un âge invraisemblablement élevé (tel que 99 ans), ce qui revient à ne pas révéler son âge effectif. Un autre usage, beaucoup plus courant, consiste à modifier sa date de naissance pour se vieillir ou, plus souvent, pour se rajeunir. Cette pratique est apparente sur Meetic où les années de naissance des utilisateurs connaissent des pics aux chiffres « ronds » tels que 1970, 1975 et 1980. La pyramide des âges prend ainsi une forme irrégulière et ce pour les deux sexes: le degré de surreprésentation des inscrits aux chiffres ronds des années de naissance est semblable pour les femmes et les hommes (graphique 1).

Graphique 1 : Pyramide des âges déclarés sur Meetic.fr en 2014

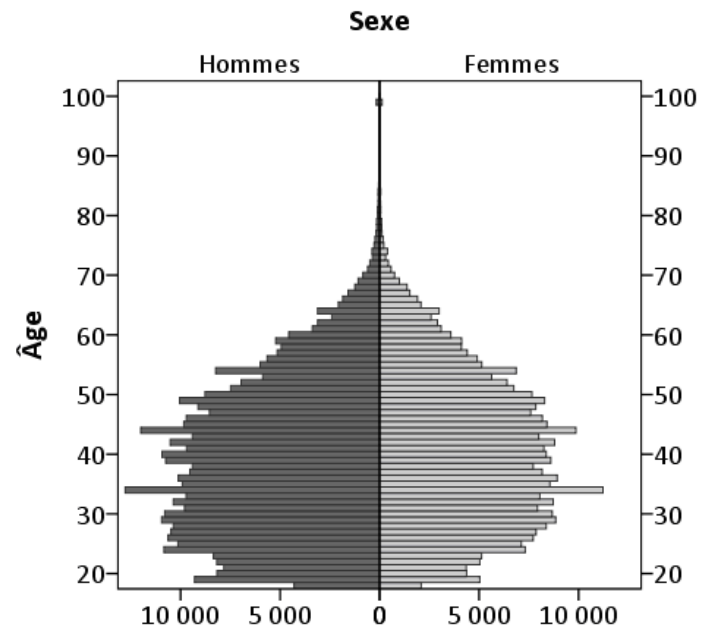

Lecture : en 2014, 12784 comptes d'utilisateurs d'hommes de 34 ans (nés en 1980) sont créés sur Meetic.fr contre 9918 comptes d'utilisateurs d'hommes de 35 ans (nés en 1979). La même année, 11232 comptes d'utilisatrices de femmes de 34 ans (nées en 1980) sont créés contre 8564 comptes d'utilisatrices de femmes de 35 ans (nées en 1979).

Champ : comptes d'utilisateurs «actifs » enregistrés sur le site Meetic.fr en 2014. Par utilisateur « actif » nous entendons un utilisateur qui s'est reconnecté au site au moins un jour après la date de création du compte $(\mathrm{N}: 988$ 883).

Source: Base d'utilisateurs de Meetic.fr, 2014, Meetic Group. 
Alors que les utilisateurs de sites de rencontres se voient souvent imputés des comportements mensongers en général, notre enquête indique comme d'autres que les ruses sont en réalité limitées et concernent tout particulièrement l'âge, la taille et le poids ${ }^{16}$. Ces différentes caractéristiques ont en commun de former un continuum. Contrairement à d'autres marqueurs de l'identité (tels que la situation matrimoniale, le niveau de diplôme ou la profession), le passage d'une catégorie à l'autre ne traduit pas en soi un changement d'état ou de statut. L'âge, la taille et le poids sont des valeurs d'échelles qui sont vouées à évoluer. Surtout, ils sont rarement appréhendés en termes objectifs mais font l'objet d'une appréciation qualitative et approximative : quelqu'un est jeune, grand ou mince. Le trucage de ces valeurs peut ainsi être compris comme une réaction à leur caractère arbitraire : l'objectivation chiffrée traduit parfois mal la manière dont ces caractéristiques sont vécues subjectivement. Plutôt qu'un comportement mensonger, la révision de l'autoportrait apparaît alors comme une manière de faire correspondre les valeurs objectives à l'expérience sociale de son âge ou de son apparence physique. De telles stratégies étaient déjà visibles dans les annonces matrimoniales où l'âge civil était souvent complété par un jugement qualitatif tel que « 49 faisant 40 », « 43 ans, physique et caractère jeunes », « 69 ans paraissant moins $»^{17}$. Ces stratégies de présentation de soi, qui consistent à faire apparaitre l'âge physique et social à côté de l'âge chronologique, sont impossibles sur les sites de rencontres qui requièrent nécessairement une date de naissance. Modifiant cette dernière, les usagers «mentent » quant à leur âge objectif pour rester fidèles à un âge subjectif qui leur parait plus favorable pour réaliser des rencontres amoureuses et sexuelles.

Les usages détournés de l'âge sur les sites de rencontres répondent aussi à l'anticipation des préférences d'âge du sexe opposé. Comme nous l'avons indiqué, les utilisateurs ont recours à des moteurs de recherche pour délimiter le vivier des partenaires potentiels. Conscients que l'âge constitue un critère central lors de ce tri, certains usagers modifient leur âge pour correspondre aux critères qu'ils

\footnotetext{
16 Brym \& Lenton 2001 ; Toma, Hancock \& Ellison 2008.

17 Fages $1972: 92$.
} 
anticipent être ceux des personnes qu'ils aimeraient rencontrer. Cette stratégie amène les deux sexes à se rajeunir. Comme le montre une étude étatsunienne, les hommes se font plus jeunes pour être éligibles par des femmes encore plus jeunes. Anticipatrices de cette préférence masculine, les femmes se rajeunissent aussi mais dans l'objectif de plaire aux personnes proches en âge ${ }^{18}$. La même tendance s'observe dans notre enquête par entretiens.

\section{[Véronique, 68 ans, chef d'entreprise à la retraite, sans enfant]}

[Relance] Donc, vous n'aviez,pas mis votre vrai âge dans le profil?

Non, pour Jean [partenaire actuel] je l'avais pas mis. J'avais pas mis mon âge, je m'étais rajeunie de six ans. C'est Liliane qui m'a dit de me rajeunir! Elle m'avait dit «mais écoute». C'est vrai que quand tu mets 58 ans... J'avais mis 53 je pense.

\section{Etpourquoi?}

Parce que Liliane m'avait dit «écoute, tu fais 50 ». J'avais mis 53 [rire]. Pourquoi ? Pour éviter la barrière tu vois. Parce que Jean, si j’avais mis mon âge, il aurait même pas répondu. C'est ce que je lui ai dit d'ailleurs. J'ai dit « écoute, tu es bien content qu'on soit ensemble et tout. Tu trouves pas que je suis... Mais si j'avais mis mon âge?» Il m'a dit «ouais ça aurait été... ». Il s'était fixé dans sa tête qu'il aimerait rencontrer, d'ailleurs je crois que c'est ce qu'il avait écrit [dans le profil], qu'il aimerait rencontrer une femme de, je sais pas, lui il faisait 63, alors il fixait 45-50 ans quoi [...] Je voulais rencontrer des gens de mon âge et pour rencontrer des gens de mon âge, il fallait que je me montre plus jeune. Sinon j'en rencontrais pas. C'est un peu ça que je tirais comme leçon de mon annonce.

Le récit de l'enquêtée illustre les stratégies de contournement de l'âge chronologique imposé par les sites de rencontres. Véronique modifie sa date de naissance pour la faire correspondre à l'âge vécu et l'âge perçu par l'entourage. Elle privilégie, non pas l'âge qu'elle a, mais celui qu'elle fait. L'objectif est notamment de rentrer dans les critères des hommes sur lesquels portent ses propres préférences. Il s'agit en effet d'" éviter la barrière » des limites d'âge supérieures que posent les hommes de son âge et qui la placent du côté des exclues. Tel que le raconte l'enquêtée, le «mensonge » n'en est pas vraiment un et n'est pas perçu comme tel par le partenaire. Face à l'autre, c'est bien

18 Ellison, Heino \& Gibbs $2006: 427$. 
l'âge social qui importe et qui finalement l'emporte dans la formation du couple.

Donnant la première place à l'âge civil, les sites de rencontres révèlent son rôle en réalité secondaire dans les rencontres ordinaires. Cet effet de miroir inversé fonctionne également pour les attentes visà-vis du partenaire. Si les sites renforcent indéniablement la place de l'âge chronologique dans la sélection amoureuse, les utilisateurs n'évaluent pas pour autant les candidats à l'aune de ce seul critère. Comme les pratiques de présentation de soi, les préférences d'âge font référence à l'identité sociale des acteurs et sont ainsi révélatrices de la signification accordée à cette caractéristique du partenaire.

\section{Les préférences amoureuses et les significations sexuées de l'âge}

Le rôle de l'âge dans le choix du conjoint a fait l'objet de nombreuses études consacrées plus spécifiquement à l'écart d'âge entre partenaires. Le constat de départ est celui d'une asymétrie structurelle des couples hétérosexuels : dans la majorité des unions, la femme est plus jeune que l'homme. Cette tendance est étonnamment constante au fil du temps et à travers l'espace. Seule l'amplitude de la différence d'âge semble en effet différencier les pays et les époques ${ }^{19}$. Ainsi, de nombreux auteurs y voient une norme culturelle universelle qui voudrait qu'en tout temps et en tous lieux, « les hommes préfèrent les femmes jeunes et que les femmes préfèrent les hommes d'âge mûr $»^{20}$. Cette lecture naturalisante des préférences d'âge n'est pas sans poser problème. D'abord, elle donne le primat à l'âge civil dans l'évaluation des partenaires. Ensuite, elle suppose, sur la base de l'écart d'âge moyen des conjoints, une préférence univoque des femmes et des hommes pour une telle asymétrie. L'étude des usages des sites de rencontres va à l'encontre de ces deux hypothèses. Elle

19 En France, la différence d'âge des conjoints s'est réduite au cours du siècle dernier sans pour autant disparaître. Elle était en moyenne de 2,3 ans pour les couples formés dans les années 1990. Pendant cette même période, seulement $16 \%$ des unions comptaient une femme plus âgée que son partenaire. Vanderschelden 2006.

20 Mignot $2010: 316$. 
indique que l'âge n'a pas le même rôle dans le choix du partenaire pour les deux sexes et que sa signification et son importance varient au fil de la vie. Aussi, si l'écart d'âge en faveur de l'homme est une tendance forte, il n'est pas pour autant unanimement sollicité par les femmes et les hommes. Par le biais des préférences d'âge exprimées par les utilisateurs de ces services, on revient donc sur la place de l'âge dans la sélection des partenaires et les attitudes des deux sexes envers la différence d'âge au sein du couple.

L'analyse des critères d'âge renseignés dans les profils d'utilisateurs du site Meetic permet d'établir les grandes tendances quant aux préférences des femmes et des hommes vis-à-vis de l'âge du futur partenaire. Le premier constat est que l'éventail des âges envisagés est relativement large : une majorité des utilisateurs affichent une tranche d'âge égale ou supérieure à 10 ans (64\%), et ceux qui indiquent un intervalle étroit, de trois ans ou moins, sont très peu nombreux $(5 \%)$. Qu'ils aient ou non un âge idéal, les candidats à la rencontre font donc preuve de seuils d'admissibilité plus généreux. Le degré de générosité est cependant variable : à presque tous les âges, les femmes indiquent des tranches d'âges plus resserrées que ne le font les hommes. Surtout, les deux sexes diffèrent quant à la nature des préférences déclarées, acceptant plus ou moins facilement la perspective d'un partenaire plus jeune ou plus âgé. On détaille alors ces positions clivées envers l'écart d'âge des conjoints, en s'intéressant d'abord aux préférences des jeunes en début de parcours affectif, et ensuite à celles des personnes plus âgées ayant vécu une rupture. La mise en perspective de ces deux groupes donne à voir les enjeux différents associés à l'âge du partenaire selon les sexes et le moment de la vie.

\section{Les désirs de féminité et de maturité en début de parcours amoureux}

Parmi les utilisateurs de Meetic, les jeunes se distinguent nettement des autres quant aux critères d'âge qu'ils affichent dans leur profil. D'une part, leurs préférences tranchent avec celles des femmes et des hommes plus âgés. D’autre part, c'est chez les plus jeunes que les désirs sont les plus contrastés entre les deux sexes. Ainsi, les hommes de 18 ans à 24 ans se montrent relativement indifférents vis-à-vis de l'âge de leur partenaire (tableau 1). S'ils préfèrent rencontrer une femme proche en âge, ils sollicitent des personnes plus jeunes (69\%) comme des 
personnes plus âgées (95\%). Cette ouverture des possibles les spécifie vis-à-vis des autres hommes qui manifestent un intérêt plus net pour des partenaires plus jeunes. Elle les distingue également de leurs paires féminines. Les femmes de moins de 25 ans ont en effet les préférences d'âge les plus rigides. Elles expriment un désir quasiment unanime pour un partenaire plus âgé (98\%) dont le corollaire est une grande réserve vis-à-vis des hommes plus jeunes : seulement un peu plus d'un quart $(26 \%)$ envisage une relation où l'homme pourrait être le cadet. En début de parcours amoureux et sexuel, les femmes plaident donc avec force pour un écart d'âge en faveur du partenaire masculin alors que les hommes se montrent ouverts aux deux scénarios.

L'analyse des annonces publiées par de jeunes utilisateurs et utilisatrices permet d'inscrire ces préférences d'âge dans le cadre plus large de leurs attentes vis-à-vis du futur partenaire ${ }^{21}$. Elle montre d'abord l'attention portée par les jeunes hommes à la sensibilité, la douceur et la gentillesse de la partenaire, de même que son caractère sexy, féminin et tendre. Ce sont là autant de qualités traditionnellement considérées comme caractéristiques des femmes. Dans les annonces des jeunes hommes, l'accent est en effet mis sur la féminité. Si celle-ci est habituellement associée à la jeunesse, elle n'est pas le privilège des femmes plus jeunes: inscrite dans un rapport de genre, elle est moins associée à un rapport d'âge. Aussi, les utilisateurs recherchent-ils autant une femme qu'une fille alors que les utilisatrices sollicitent beaucoup plus souvent un bomme qu'un mec ou un garçon.

Les annonces féminines sont, elles, d'une autre nature. Les utilisatrices décrivent le souhait de rencontrer un partenaire attentionné, affectueux, à l'écoute, gentleman et respectueux. Elles mettent l'accent sur des qualités relationnelles qui placent l'homme dans la position de pourvoyeur d'une série de dons et de gratifications: la galanterie, l'attention, l'affection, le respect et le soin. Les utilisatrices dressent ainsi le portrait d'un homme qui les protège et sur lequel elles se reposent. À cela s'ajoute le désir d'un partenaire mûr, sérieux et posé. L'attention et le soutien, les femmes les cherchent en effet chez un homme mature.

21 Cette étude repose sur une analyse textuelle de 300 annonces publiées par des utilisateurs de Match.com âgés de 25 ans. On présente ici les mots caractéristiques des annonces féminines et masculines. 
Tableau 1. Préférences d'âge déclarées sur Meetic.fr selon le sexe et l'âge (\%)

\begin{tabular}{|l|c|c|c|c|}
\cline { 2 - 5 } \multicolumn{1}{c|}{} & $\begin{array}{c}\text { Ouvert à } \\
\text { des } \\
\text { partenaires } \\
\text { plus jeunes }\end{array}$ & $\begin{array}{c}\text { Préférence } \\
\text { exclusive pour } \\
\text { des partenaires } \\
\text { plus jeunes }\end{array}$ & $\begin{array}{c}\text { Ouvert à des } \\
\text { partenaires } \\
\text { plus âgés }\end{array}$ & $\begin{array}{c}\text { Préférence } \\
\text { exclusive } \\
\text { pour des } \\
\text { partenaires } \\
\text { plus âgés }\end{array}$ \\
\hline $18-24$ ans & 26 & 1 & 98 & 42 \\
\hline $25-34$ ans & 62 & 1 & 99 & 21 \\
\hline $35-39$ ans & 69 & 1 & 98 & 19 \\
\hline $40-49$ ans & 80 & 5 & 91 & 10 \\
\hline $50-59$ ans & 84 & 12 & 84 & 7 \\
\hline 60 ans et + & 89 & 18 & 75 & 4 \\
\hline Total & 69 & 5 & 92 & 17 \\
\hline Hommes & & & & 12 \\
\hline $18-24$ ans & 69 & 1 & 95 & 1 \\
\hline $25-34$ ans & 96 & 4 & 92 & 1 \\
\hline $35-39$ ans & 98 & 9 & 85 & 1 \\
\hline $40-49$ ans & 98 & 21 & 70 & 1 \\
\hline $50-59$ ans & 99 & 38 & 53 & 3 \\
\hline 60 ans et + & 99 & 51 & 38 & \\
\hline Total & 93 & 17 & 77 & \\
\hline
\end{tabular}

Lecture : $26 \%$ des femmes de 18-24 ans indiquent une tranche d'âge ouverte à des partenaires plus jeunes (l'âge minimum renseigné est inférieur à l'âge propre) contre $69 \%$ des hommes de même âge. 18\% des femmes de 60 ans et plus indiquent une tranche d'âge exclusivement centrée sur des partenaires plus jeunes (l'âge maximum est inférieur à l'âge propre) contre $51 \%$ des hommes de même âge.

Champ : comptes d'utilisateurs "actifs» enregistrés sur le site Meetic.fr en 2014. Par utilisateur « actif » nous entendons un utilisateur qui s'est reconnecté au site au moins un jour après la date de création du compte ( $\mathrm{N}: 988$ 883).

Source: Base d'utilisateurs de Meetic.fr, 2014, Meetic Group. 
Les préférences d'âge déclarées par les utilisatrices doivent être comprises à la lumière de cette recherche de maturité chez le partenaire masculin que Michel Bozon avait constatée chez les jeunes femmes au milieu des années 1980. L'auteur montre comment il traduit la valorisation de l'expérience de l'homme et plus précisément son installation sociale. Ainsi, la maturité du partenaire renvoie moins à son âge chronologique qu'à son âge social : est mature un homme qui fait preuve d'indépendance et d'une situation stable ${ }^{22}$. Depuis 30 ans, les aspirations amoureuses des jeunes femmes semblent avoir peu changé. La préférence pour un partenaire ayant délaissé l'incertitude et l'insouciance de la jeunesse se lit dans les annonces sur les sites de rencontres mais apparaît aussi dans les récits des jeunes enquêtées.

\section{[Pauline, 22 ans, étudiante salariée, sans enfants]}

Il [partenaire plus âgé] a quand même une expérience de la vie qui fait que... il fait pas partie du nombre de mecs qui savent pas ce qu'ils veulent. En gros, entre Anthony et Clément [partenaires précédents], ça a été ça quoi. Enfin, concrètement, c'était parce qu'ils étaient trop jeunes et qu'ils ne savaient pas, ni qui ils étaient, ni où ils allaient, ni pourquoi. Enfin voilà. Et donc, j'ai un peu marre de ce truc-là.

Si plusieurs des jeunes enquêtées ont connu des partenaires sexuels de même âge, leurs expériences de couple concernent plus souvent des hommes plus âgés qu'elles. Lorsqu'elles entrent dans la conjugalité, leur choix portent avant tout sur des hommes dont l'expérience de vie témoigne de «qui ils sont» et « où ils vont» pour emprunter les termes de l'enquêtée citée ci-dessus. Ce sont des hommes qui, contrairement aux pairs, rassurent par leur stabilité sociale et qui par conséquent peuvent apporter de la sécurité, y compris affective. L'allongement de la scolarité des femmes, de même que leur plus grand accès au marché du travail et aux postes de responsabilité, ne semblent pas avoir modifié sensiblement la manière dont celles-ci se représentent la relation conjugale et leur place au sein du couple. Bien que les femmes se soient élevées dans la hiérarchie sociale, elles paraissent réticentes à transférer l'indépendance acquise dans le domaine amoureux. Par leur définition de la conjugalité, les jeunes femmes portent en effet leurs choix sur des partenaires qui les

22 Bozon 1990a et 1990b. 
précèdent sur le plan scolaire et professionnel ${ }^{23}$. En découle une asymétrie des âges, exprimant l'asymétrie des statuts des deux partenaires, dont les jeunes femmes sont les principales avocates.

\section{Les âges sexués de la séparation}

Avec l'avancée en âge, les préférences amoureuses des femmes et des hommes non seulement changent mais s'inversent par rapport aux positions tenues par les jeunes. Progressivement, les femmes se montrent de plus en plus indifférentes vis-à-vis de l'âge du futur partenaire : entre 40 ans et 49 ans, elles demandent à rencontrer des hommes plus jeunes $(80 \%)$ comme des hommes plus âgés (91\%). Les hommes apparaissent en revanche de plus en plus sélectifs. La part d'utilisateurs qui sollicitent exclusivement des femmes plus jeunes va notamment croissant avec l'âge : c'est le cas de $21 \%$ des hommes âgés de 40 ans à 49 ans et $51 \%$ de ceux de 60 ans et plus (tableau 1). Ainsi, les attitudes des deux sexes envers l'écart d'âge traditionnel des partenaires se sont inversées. Ce résultat contraste avec les observations faites dans les années 1980 qui montraient que, à tout âge, les hommes envisageaient plus facilement que les femmes une relation dans laquelle l'homme serait plus jeune ${ }^{24}$. L'étude des préférences déclarées sur Meetic indique au contraire que, à partir de 40 ans, les hommes sont plus demandeurs que ne le sont les femmes d'un écart d'âge en leur faveur. Cette recomposition des préférences amoureuses doit se lire à la lumière des parcours conjugaux qui se sont complexifiés. Les utilisateurs de Meetic, âgés de 40 ans et plus, s'y sont pour la plupart inscrits suite à une rupture. Leurs préférences d'âge s'éclairent alors par les aspirations différentes que fait naitre l'expérience de la séparation chez les deux sexes.

Des considérations spécifiques entrent en effet en ligne de compte lorsque les individus ont déjà vécu en couple. En début de parcours conjugal, et comme en témoignent les annonces publiées par les jeunes utilisateurs de sites de rencontres, les attentes vis-à-vis du partenaire portent essentiellement sur ses qualités et sa "personne». Avec l'avancée en âge et les expériences amoureuses qui en

\footnotetext{
23 Bozon 1990a et 1990b.

24 Bozon 1990a : 346-347.
} 
découlent, le partenaire est aussi jugé en fonction de sa situation conjugale et familiale.

\section{[Cécilia, 39 ans, secrétaire, deux enfants en bas âge]}

Vous aviez. une idée de qui vous vouliez, rencontrer [sur Meetic]?

C'est pas que j'avais une idée, c'était que je savais ce que je voulais pas en tout cas. A partir de là... Moi, je voulais quelqu'un avec qui je pouvais partager certaines choses, quelqu'un qui m'attire, de classe moyenne, avec une situation [matrimoniale] claire. J'avais pas envie de me retrouver avec quelqu'un, après c'est très égoïste parce que moi j’ai des enfants, mais moi j'ai pas envie de me retrouver un weekend sur deux avec cinq gamins quoi. Ça, ça m’est très difficile à envisager. Je veux pas de conflits non plus. Parce que forcément, quand on a 40 ans on va forcément tomber sur un homme qui est séparé. Donc il a déjà une vie antérieure, il a une ex-femme, il a des enfants, il faut faire avec tout ça [...] Donc lui ça tombait bien parce qu'il avait 47 ans, trois enfants dont deux qui ne vivent plus au domicile et qui travaillent et le dernier qui a 15, 16 ans. L’homme idéal quoi.

Invitée à décrire le partenaire souhaité, l'enquêtée en fait un portrait par la négative qui privilégie les critères d'élimination aux critères de sélection. Elle évoque succinctement ce qu'elle attend, pour s'attarder plus longuement sur ce qu'elle ne souhaite pas: un homme avec enfants et un passé conjugal toujours présent. Ayant vécu une déception amoureuse, et avec deux enfants en bas âge à charge, elle aborde la vie conjugale par ses aspects pratiques plutôt que ses dimensions affectives. Dans sa forme et son contenu, le récit est teinté par un certain pragmatisme caractéristique des entretiens avec des femmes ayant déjà connu une vie de couple et de famille. Ce pragmatisme caractérise également les préférences d'âge. Si l'homme de 47 ans décrit en entretien est «idéal », c'est qu'il n'a plus d'enfants en bas âge. Réticente à une recomposition familiale, l'enquêtée souhaite rencontrer un partenaire "sans passé » ou avec un passé conjugal lointain. L'âge de l'homme paraît ici comme un critère indirect et plus précisément comme une fonction de l'âge de ses enfants. L'indifférence relative que manifestent, lorsqu'elles sont d'âge moyen, les utilisatrices de Meetic à l'égard de l'âge du partenaire peut se comprendre à la lumière de ces considérations : à un moment de la vie où la rencontre amoureuse traduit une remise en couple, 
l'âge compte moins en tant que tel que la situation familiale à laquelle il se voit associé.

De même, la volonté des hommes de rencontrer une partenaire plus jeune ne doit pas être interprétée seulement comme un désir de jeunesse. Elle peut aussi se comprendre, comme pour les femmes, à la lumière de leur trajectoire conjugale et familiale et de considérations relatives à la trajectoire de la partenaire. Les hommes entrent plus tardivement en couple que ne le font les femmes et se trouvent, après une séparation, moins souvent avec des enfants à charge ${ }^{25}$. ̀̀ "héritage » inégal après une rupture, les deux sexes n'ont pas les mêmes ambitions concernant la nouvelle relation et, plus précisément, n'ont pas les mêmes projets parentaux. Cela ressort notamment des projets d'avenir renseignés dans les profils de Meetic. À la question de savoir si l'on «veut des enfants», les femmes sont plus nombreuses que les hommes à répondre par la négative. Si une majorité des personnes des deux sexes désirent, à des taux semblables, être parents lorsqu'ils sont jeunes, l'écart se creuse à partir de 35 ans avec les femmes qui refusent plus fermement cette perspective $^{26}$. La préférence des hommes pour des femmes plus jeunes peut alors être comprise comme une manière d'approcher des personnes a priori moins marquées par un passé familial et conjugal antérieur. Elle traduirait le désir de recommencer avec une partenaire tout aussi disponible pour une nouvelle vie de famille et de couple. Ce désir se lit notamment dans le récit de Bruno qui a rencontré sur Meetic une femme, plus jeune, avec qui il souhaite refonder une famille. Père, il ne vit pas avec ses enfants et prévoit, au moment de l'entretien, d'aménager à Paris avec sa nouvelle partenaire. À ce sujet il ajoute :

25 Chardon, Daguet \& Vivas 2008.

26 Entre 35 ans et 39 ans, 22\% des utilisatrices déclarent ne pas vouloir avoir d'enfants contre $10 \%$ des utilisateurs. Entre 40 ans et 44 ans, elles sont $52 \%$ à rejeter cette perspective contre $22 \%$ des hommes. Un rapport différencié aux projets conjugaux et familiaux s'observe aussi dans les réponses à la question de la « vision du mariage » posée dans le profil de Meetic. À âge égal, les femmes sont plus nombreuses que les hommes à rejeter la perspective du (re)mariage. Source : Base d'utilisateurs de Meetic.fr, 2014, Meetic Group. Champ: comptes d'utilisateurs « actifs » enregistrés sur le site Meetic.fr en 2014 (N : 988 883). 


\section{[Bruno, 44 ans, soudeur intérimaire, deux enfants adolescents]}

Le premier truc qu'elle [partenaire actuelle] m'avait dit quand on s'est rencontrés sur Meetic, c'était que j’habite loin. Ça peut poser problème. Et je lui avais dit « oui effectivement, au début ça peut être un peu compliqué mais après il faut voir, si on s'entend, s'il y a un bon feeling... » Je sais que la maison où je suis, c'est ma maison, je l'ai retapé, mais il y a rien qui me retient là-bas [...] Pour l'amour, j’aurais même pu traverser les océans. Même si effectivement j'ai deux enfants qui sont là, qui sont sur Troyes, qui ont 13 et 15 ans maintenant. Mais ça, pour moi, ça sera plus un frein.

Divorcé, Bruno souhaite repartir de zéro et se sent libre pour le faire. Cette table rase de la première union est moins accessible aux femmes de son âge qui en portent plus souvent les conséquences, c'est-à-dire, qui ont la principale responsabilité des enfants. Les préférences d'âge des hommes et des femmes s'éclairent donc par les enjeux différents associés à la remise en couple pour les deux sexes. Le rapport à l'âge du partenaire n'est pas le même notamment parce que les ambitions et les marges de manœuvre sont inégales quand il s'agit de « refaire sa vie». Ainsi, lorsque leurs situations se rapprochent, il en va également des préférences. C'est ce qu'indique le récit de Michèle qui, célibataire et sans enfant, fait part de critères d'âge proche des hommes de son âge.

\section{[Michèle, 49 ans, sans activité professionnelle (écrivaine), sans enfant]}

J'étais déjà au milieu de la quarantaine et, à cet âge-là, je ne pense pas que le mariage soit important. Et je voulais pas d'enfants et je voulais pas être avec quelqu'un qui avait des enfants. Je me sentais très au clair sur ça. Ce que je voulais c'était rencontrer quelqu'un, m'engager, pour voyager ensemble, pour faire des choses intéressantes ensemble, quelqu'un qui serait dans la même phase que moi et puis, juste profiter de la vie quoi $[\ldots]$ Je me suis dit, je mets 35 comme le plus bas et puis 50 comme l'âge maximum. C'était la limite. Mais j’avais déjà du mal avec ça. Je regardais les photos d'hommes dans la cinquantaine et ils avaient l'air si vieux! Et je ne pouvais tout simplement pas être attirée par ça. Pas à ce stade de ma vie. Donc la gamme était plus large en dessous de mon âge qu'au-dessus.

Désireuse de vivre une nouvelle histoire d'amour, l'enquêtée s'inscrit sur Meetic en 2007. Âgée alors de 46 ans, elle souhaite renouer avec la conjugalité mais rejette la perspective de fonder une famille ou de se marier - événements qu'elle ne considère plus d'actualité à son âge. Elle voudrait ainsi rencontrer un homme qui partage son mode de vie et ses projets: quelqu'un sans enfants, libre de voyager et de 
"profiter de la vie». De manière analogue aux hommes de son âge - cherchant chez les femmes plus jeunes des partenaires susceptibles de partager leurs ambitions conjugales et familiales -, Michèle se tourne vers des hommes plus jeunes pour trouver quelqu'un qui est dans « la même phase qu'[elle]». Son récit souligne l'imbrication des préférences d'âge dans les considérations du mode de vie et de la situation familiale du partenaire. Mais il montre aussi comment le statut marital et parental influence la manière d'appréhender son âge propre. De par sa situation, caractéristique des jeunes, Michèle se sent elle aussi « jeune ». En même temps, elle trouve « vieux» les hommes plus âgés qu'elle et dit ne pas être « attirée par ça. Pas à ce stade de [sa] vie ». La préférence pour des partenaires plus jeunes reflète ici le sentiment d'être soi-même jeune. La même chose peut se lire dans le récit de Bruno qui explique son désir de refonder une famille à la lumière de sa jeunesse: "je suis encore jeune et donc je me dis, pourquoi pas un autre enfant quoi ». Les entretiens soulignent que les critères d'âge ne sont pas seulement guidés par l'âge civil des acteurs mais aussi leur âge social, défini par les conditions de vie, les étapes franchies et le sentiment subjectif d'où l'on se trouve dans la vie ${ }^{27}$. Cet âge vécu n'est pas le même pour les hommes et les femmes après une séparation. Moins marqués par les premières unions, les hommes sont « encore jeunes » et se tournent alors vers des partenaires qu'ils identifient comme l'étant aussi. La même chose s'observe chez des femmes qui, en raison d'un parcours inhabituel, se perçoivent plus jeunes que leurs paires avec enfants.

\section{Les usages de l'âge et les inégalités de genre}

Dans la formation des couples, l'âge prend des sens différents. Les sites de rencontres le montrent d'autant mieux qu'ils tentent de réduire cette propriété sociale à une valeur objective. Plus encore, ces services mettent en exergue les attitudes contrastées des femmes et des hommes à l'égard de leur âge comme à celui du partenaire. Ce faisant, ils révèlent l'inégalité de genre qui se loge dans la conjugalité.

27 Bourdieu 1980 ; Bozon 1990b. 
D'abord, les préférences d'âge des jeunes utilisateurs témoignent d'un rapport dissymétrique des deux sexes à l'expérience amoureuse. Contrairement aux hommes, les jeunes femmes font preuve de ce que Sonia Dayan-Herzbrun appelle une « dépendance affective » vis-à-vis du partenaire ${ }^{28}$. Plus que la réciprocité, elles cherchent dans le conjoint une source de soutien et de sécurité et se tournent pour cela vers des hommes plus âgés. L'indépendance économique acquise par les femmes au cours des dernières décennies ne semble pas avoir effacé cette dépendance émotionnelle. Le couple apparaît au contraire comme un lieu où l'asymétrie des sexes persiste avec une force singulière.

La situation des personnes séparées révèle, elle, les implications inégales de la vie conjugale et familiale pour les deux sexes. Parce que ce sont largement les femmes qui ont la garde des enfants après une rupture, les hommes ont plus de marge de manœuvre pour « refaire leur vie». Aussi, la séparation rend les hommes jeunes de nouveau. Célibataires et sans enfants à charge, ils sont prêts pour un nouveau départ et sollicitent des femmes qui, jeunes "également», sont susceptibles de partager leurs ambitions. Ainsi, l'avancée en âge - et les expériences amoureuses et parentales qui en découlent - marque davantage les femmes que les hommes. Cela participe à l'exclusion des femmes âgées qui, tout aussi touchées par les séparations que leurs pairs masculins, peinent cependant à se remettre en couple. Les usages de l'âge sur les sites de rencontres témoignent donc des conditions inégales dans lesquelles hommes et femmes entrent dans la conjugalité, tout comme les perspectives différentes qui s'ouvrent à eux lorsqu'ils en sortent.

\section{Bibliographie}

Bergström Marie, 2014, «Au bonheur des rencontres. Classe, sexualité et rapports de genre dans la production et l'usage des sites de rencontres en France », thèse de doctorat, SciencesPo Paris.

Bourdieu Pierre, 1980, Questions de sociologie, Paris, Éditions de Minuit.

28 Dayan-Herzbrun $1982: 120$. 
Bozon Michel, 1990a, «Les femmes et l'écart d'âge entre conjoints : une domination consentie. I. Types d'union et attentes en matière d'écart d'âge », Population, $\mathrm{XLV} / 2$, p. 327-360.

—, 1990b, «Les femmes et l'écart d'âge entre conjoints : une domination consentie. II. Modes d'entrée dans la vie adulte et représentations du conjoint », Population, $\mathrm{XLV} / 3$, p. 565-602.

—, 2008, "Pratiques et rencontres sexuelles : un répertoire qui s'élargit », in Nathalie BAjos \& Michel Bozon (dir.), Enquête sur la sexualité en France. Pratiques, genre et santé, Paris, La Découverte, p. 273-296.

BRYM Robert J. \& Rhonda L. LENTON, 2001, « Love online: a report on digital dating in Canada », Toronto, MSN.CA.

Chardon Olivier, Daguet Fabienne \& Émilie Vivas, 2008, «Les familles monoparentales. Des difficultés à travailler et à se loger », Insee Première, 1195, p. 1-4.

CHAulet Johann, 2009, «Sélection, appariement et modes d'engagement dans les sites de mise en relation », Réseaux, II/154, p. 131-164.

DAYAN-Herzbrun Sonia, 1982, «Production du sentiment amoureux et travail des femmes », Cahiers internationaux de Sociologie, LXXII/72, p. 113-130.

Ellison Nicole B., HeIno Rebecca \& Jennifer GIBBS, 2006, « Managing impressions online: self-presentation processes in the online dating environment », Journal of Computer-Mediated Communication, XI/2, p. 415-441.

Eymard-Duvernay François \& Emmanuelle MARChal, 1997, Façons de recruter : le jugement des compétences sur le marché du travail, Paris, Métailié.

—, 2000, «Qui calcule trop finit par déraisonner : les experts du marché du travail », Sociologie du travail, XLII/3, p. 411-432.

FAGES Jean-Baptiste, 1972, Miroirs de la société. II. Les petites annonces, Paris, Mame.

GARDEN Maurice, 2008 [1981], «Les annonces matrimoniales dans la lunette de l'historien ", in Un bistorien dans la ville, Paris, Éditions de la Maison des sciences de l'homme, p. 285-298.

KALIFA Dominique, 2011, "L'invention des agences matrimoniales », L'Histoire, 365, p. 76-79.

MARTIN Marc, 1980, "Images du mari et de la femme au XXe siècle, les annonces de mariage du "Chasseur français" ", Revue d'histoire moderne et contemporaine, XXVII/2, p. 295-311.

Mignot Jean-François, 2010, «L'écart d'âge entre conjoints », Revue française de sociologie, LI/2, p. 281-320.

SINGLY François de, 1984, «Les manœuvres de séduction : une analyse des annonces matrimoniales », Revue française de sociologie, XXV/4, p. 523-559. 
Toma Catalina L., Hancock Jeffrey T. \& Nicole B. Ellison, 2008, « Separating fact from fiction: an examination of deceptive self-presentation in online dating profiles », Personality and Social Psychology Bulletin, XXXIV/8, p. 1023-1036.

VANDERSChELDEN Mélanie, 2006, «L'écart d'âge entre conjoints s'est réduit », Insee première, 1073, p. 1-4.

\section{Méthodes et données}

Cet article se base sur une recherche sociologique consacrée à la production et aux usages des sites de rencontres en France. Pour étudier la place de l'âge dans les rencontres en ligne, on mobilise plus précisément trois enquêtes. La première, conduite entre 2008 et 2012, repose sur une cinquantaine d'entretiens biographiques menés avec des usagers de sites de rencontres, d'âge et de milieux sociaux diversifiés. La deuxième est une analyse de profils d'utilisateurs enregistrés sur le site Meetic.fr. Dans le cadre d'un partenariat négocié avec l'entreprise éditrice de ce site, nous avons obtenu une copie de la base d'inscrits. Celle-ci contient l'ensemble des comptes d'utilisateurs enregistrés sur la plateforme entre 2002 (l'année de son lancement) et 2014, soit plus de 16 millions d'inscriptions. On se sert de ces données pour étudier la distribution des âges au sein de la population usagère et les préférences d'âge déclarées par les utilisateurs. Ces deux types d'informations font l'objet de questions obligatoires dans le formulaire d'inscription du site : pour pouvoir accéder à la plateforme, les futurs utilisateurs doivent renseigner leur date de naissance ${ }^{29}$ et indiquer une tranche d'âge souhaitée pour les partenaires potentiels allant de 18 ans à 99 ans. Ces informations ont fait l'objet d'une analyse statistique dont on présente ici quelques-uns des résultats principaux. La troisième enquête repose enfin sur une analyse textuelle d'un corpus d'«annonces» (textes de présentation) publiées sur le site Match.com. L'étude a été conduite en 2008 et porte sur 300 annonces publiées par des jeunes utilisateurs de 25 ans. Le site Match.com a été retenu en tant que seul grand site de rencontres généraliste à rendre obligatoire à la fois le renseignement des critères d'âge et la rédaction d'une annonce d'au moins 200 caractères. Les annonces ont été analysées à l'aide du logiciel SPAD.

29 Meetic.fr comme l'écrasante majorité des sites de rencontres ciblent des personnes majeures. L'âge minimum accepté par le site est donc 18 ans et l'âge maximum 99 ans. 